\title{
Digital holographic interferometry in physical nanomeasurements
}

\author{
Z. T. Azamatov ${ }^{1}$, N. A. Akbarova ${ }^{1}$, I. A. Kulagin ${ }^{1}$, V. E. Gaponov², V. I. Redkorechev ${ }^{1}$, A. M. Isayev ${ }^{3}$ \\ ${ }^{1}$ National University of Uzbekistan, 100074, Tashkent, Uzbekistan \\ ${ }^{2}$ Kripton Ltd., Snejinsk, Russia \\ ${ }^{3}$ Center of Laser Technologies Scientific Firm, Almaty, Kazakhstan \\ ilya_kulagin@list.ru, zakir.azamatov@mail.ru,gorani@bk.ru
}

PACS 07.60.-j

DOI $10.17586 / 2220-8054-2017-8-1-79-84$

The possibility of using digital holographic interferogram obtained via laser radiation at different wavelengths and different pulse lengths in different times for the reconstruction of dynamic phase changes was explored. The opportunities for applying the digital holographic interferometry method to the study of resonant mode shapes in resonant acoustic spectroscopy are shown. The results of experimental measurements and analytical calculations are submitted.

Keywords: information transmission, digital holographic interferometry.

Received: 10 August 2016

Revised: 6 September 2016

\section{Algorithm Description}

Ranges of resonant acoustic vibrations and their form bear information on the internal structure of the studied solid. From a range of resonance frequencies of known form for a sample, the elasticity tensor can be measured and areas of localization of defects can be defined. One of the most sensitive methods for determining resonance frequencies and studying the form of resonant fluctuations is digital holographic interferometry (DHI). The method of DHI is based on comparing the phases of two object waves recovered from the digital holograms corresponding to different phases of process of a surface deflection of object. For registration of the digital hologram, the radiation of the laser is divided into the object bunch covering object, and the basic bunch which is directly arriving on a receiving sensor of a digital video camera. The object bunch covers object in the direction of $\mathbf{k}_{\mathbf{i}}$. The part of the world reflected by object in the direction of $\mathbf{k}_{\mathbf{v}}$, called 'the direction of supervision', passes through optical system of the interferometer and creates the image of object on the receiving sensor of a digital video camera. The hologram of the focused image forms on the CCD array of the digital camera as result of an interference between basic and object bunches. For allocation of measuring information, the algorithm of processing digital hologram1 was used.

Let $R(x, y)$ be a smooth basic wave, and $U(x y)$ is an object wave proceeding from object. Then, the intensity registered on the $\mathrm{CCD}$ array of a receiving video camera is described by expression:

$$
I_{H}(x, y)=\left|R_{H}(x, y)\right|^{2}+\left|U_{H}(x, y)\right|^{2}+R_{H}(x, y) U_{H}^{*}(x, y)+R_{H}^{*}(x, y) U_{H}(x, y),
$$

where, $H$ - the index designating the hologram plane, and an index ${ }^{*}$ designates complex interface. The intensity described by the equation (1) is registered on the two-dimensional electronic device consisting of ranks of the sensitive cells-pixels ( $M \times N$ cells) having the sizes $\Delta x \times \Delta y$ that allows one to write down value of intensity in the form of the IH function $(m \Delta x, n \Delta y)$ where $m$ and $n$-integers. The last two terms of Eq. (1) contain information on amplitude and phase of an object wave. By the Fourier transformation to the registered array, it is possible to allocate and filter one of two last terms of Eq. (1). After filtering and Fourier transformation return the complex amplitude of the object wave front is obtained. From the complex amplitude of UH received in a digital form ( $m \Delta x, n \Delta y)$, the phase of the wave front of an object wave can be calculated:

$$
\Phi_{n}=(m \Delta x, n \Delta y)=\operatorname{atan} \frac{I_{m}\left[U_{H}(m \Delta x, n \Delta y)\right]}{R_{m}\left[U_{H}(m \Delta x, n \Delta y)\right]} .
$$

By subtraction of the phase values for the object field, we obtain the difference of phases, which allows us to calculate the shift of points of object of $\mathbf{d}$ in the directions, giving:

$$
\Delta \Phi=\frac{2 \pi}{\lambda} \mathbf{d} \cdot \mathbf{s},
$$

where, $\lambda$ is the laser wavelength, $\mathbf{s}$ is the vector interferometer sensitivity defined by the expression $\mathbf{s}=\mathbf{k}_{\mathbf{i}}-\mathbf{k}_{\mathbf{v}}$, $\mathbf{k}_{\mathbf{i}}$ and $\mathbf{k}_{\mathbf{v}}$ are the unit vectors of illumination and observation, respectively. 


\section{Scheme of measurements, results and discussion}

\subsection{The stroboscopic-holography method}

The stroboscopic-holography method [1] was utilized for quantitative measurements of the vibrational movement fields. At the same time, the digital holograms of the vibrating object for each resonance frequency are registered by accumulation (over $1 / 10-1 / 1000 \mathrm{~s}$ ) exposures on the receiving sensor of a digital camera in the light of the short repeating impulses of laser radiation synchronized with the set phases of fluctuation processes. Comparison of the object waves' phases to those obtained from two digital stroboscopic holograms registered in different vibration phases allows one to receive the two-exposition interferogram, which is given to quantitative interpretation. Application of long pulse exposures to the principle eliminates the need for synchronization of time intervals for exhibition of the camera's receiving sensor with the process phase, permitting the use of low-frequency digital cameras for measurements and providing an increase in the data on the studied objects. Holograms registered in the laser radiation were diffracted by the optical-acoustic modulator (OAM), which for the continuous laser radiation $(\lambda=0.53 \mathrm{~mm}, 50 \mathrm{~mW})$, allocated the impulses synchronized with the chosen phase having a fluctuation of $2 \mathrm{~ms}$. The metal plate $5 \times 5 \mathrm{~cm}$ fixed in the center was object of study. The plate was excited by the piezo vibrator at frequencies up to $20 \mathrm{kHz}$. Acoustic vibrations of a plate were registered by the vibration sensor and were processed by a range analyzer for determination of resonance frequencies. Then, at the fluctuations of a resonance frequency, the phase of resonant fluctuation was chosen and the hologram was removed. Digital holograms were removed on a matrix $1000 \times 1000$ pixels with a pixel size $5 \times 5 \mathrm{~mm}$ and were processed by the program in the MATLAB-12 environment in real time. The scheme of experiment is depicted in Fig. 1.

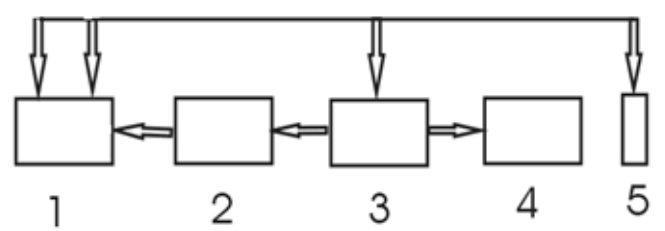

FIG. 1. Scheme of measurements: 1 - PC laptop; 2 - Interferometer in structure with the laser, the camera and OAM; 3 - Generator and analyzer of a range; 4 - A piezo vibrator and the studied sample; 5 - Sensor of vibrations

The measuring accuracy was defined by comparison with controlled movement and was $0.01 \mathrm{~mm}$. In Fig. 2 , results of quantitative measurements of the vibrational movement fields at resonance frequencies are presented. At each resonance frequency, two digital holograms corresponding to two amplitude conditions of an object in opposite phases were registered.

An upper row contained the fluctuation forms obtained by calculation by method of final elements, while an average row displayed results for the experimental determination of resonance frequencies, visualization of forms of own fluctuations and localization of nodal lines in real time.

The lower row contained results for the measurement of vibrational movement fields at resonant fluctuations. On axes $x$ and $y$-coordinates in pixels, on an axis $z$-amplitude of fluctuations in mm. The maximum amplitude of movement of points of a surface is equal to $1 \mathrm{~mm}$ and the accuracy of measured movements was $0.01 \mathrm{~mm}$.

\subsection{Dynamic phase changes in photorefractive crystals of lithium niobate}

The digital holographic interferometer (DHI) 'interferes' with the digital field derived from the actual field by digital holography (PG) [2]. These methods are based on the Huygens-Fresnel principle, the mathematical formalism of the Fourier transform, numerical algorithms for deployment phase and the digital image processing algorithms [3]. Unlike the conventional interferometer, which requires two or more coherent phase fields to obtain a phase interferogram, in the DHI, one can calculate them by obtaining the interference field incoherent phase fields (e.g. fields that existed at different times and thus cannot interfere with each other). As a result of digital 'interference' such fields produce a virtual interferogram. A necessary condition for the implementation of single-wave DHI is the appropriate smallness of the optical path difference between adjacent pixels (less than half a wavelength $(\Delta \leq \lambda / 2))$. In cases where this condition is violated, in the recovery process (deployment), an uncertainty arises in the phase field equal to modulo $2 \pi$. The conditions $\Delta \leq \lambda / 2$ puts a hard limit on the range of single wavelength measurements in the DHI in relation to the natural spatial resolution of modern radiation detectors. 


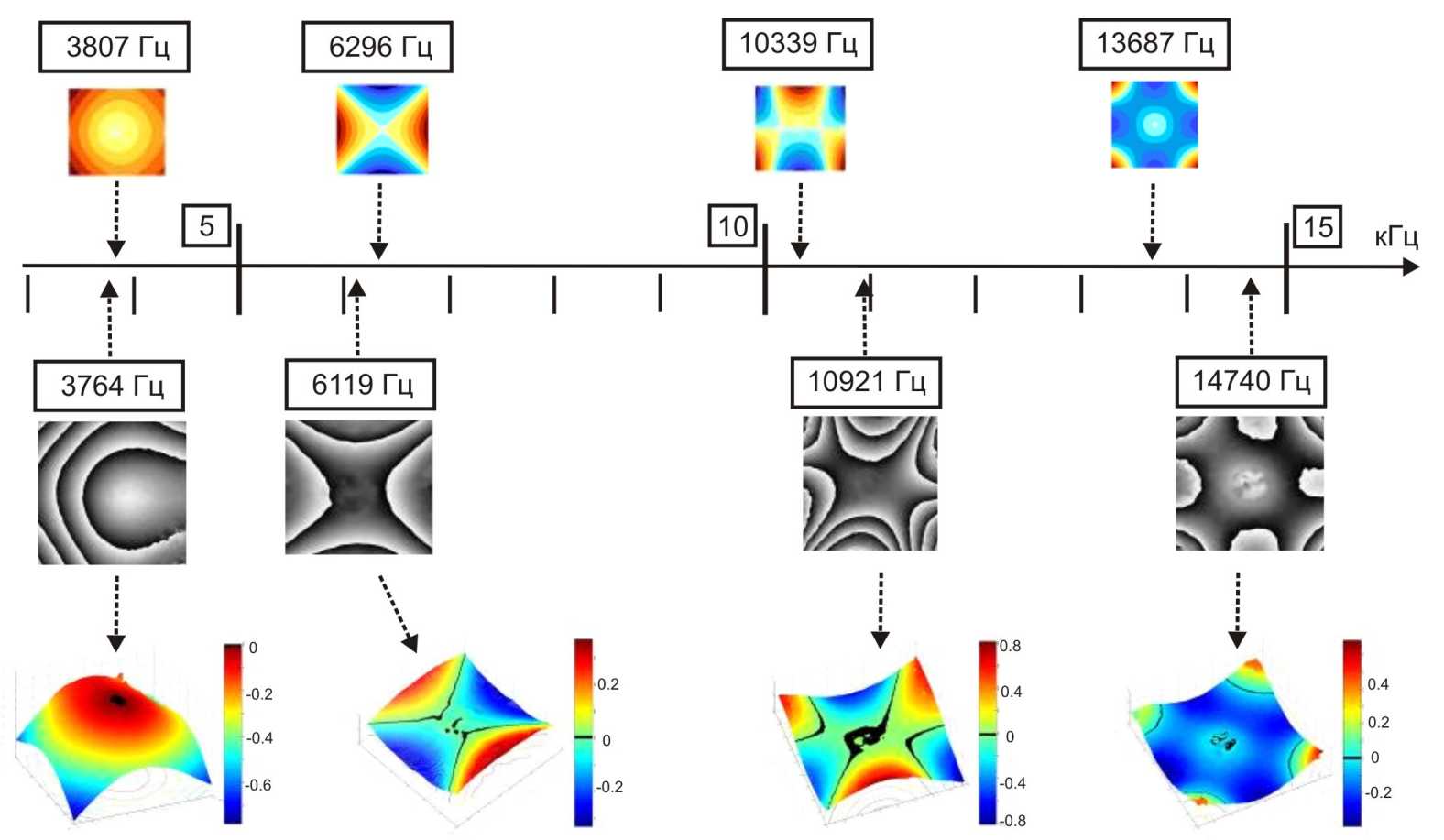

FIG. 2. The stroboscopic-holography method

One way to eliminate this uncertainty is the use of far infrared rays. However, the practical implementation of this method is limited due to the long-wave threshold of the detectors' sensitivity. Another method is the use of two waves of the visible range of different frequencies [4,5]. Two-tone CGI uses two data sets obtained at different wavelengths $\lambda_{1}$ and $\lambda_{2}$. The recovery phase of the field is carried out on an equivalent wavelength $\Lambda_{12}=\lambda_{1} \lambda_{2} /\left|\lambda_{1}-\lambda_{2}\right|$, the greater the wavelength used real light in the visible range. Thus, the use of two-tone DHI significantly softens demands on spatial resolution radiation detectors, reduces uncertainty in the recovery phase of the field and increases the sensitivity. However, enhancements in this effect also result in increased noise. In order to find an acceptable compromise, one must use different combinations of virtual and real wavelengths $\Lambda_{12}, \lambda_{1}, \lambda_{2}$ as a reference and object waves in the reconstruction phase of the interferogram. Thus, the developed technology to date information about the interference of multi-colored fields aimed at solving the problem of the uncertainty inherent in all $2 \pi$ interference measuring techniques are used as measures of real measuring radiation wavelength.

In this paper, we used two methods of digital exposure interferometry - phase difference obtaining a surface carrying information about the changes in the test object during the time between exposures. These compared hologram phase fronts were shot at different wavelengths in pulses of varying duration. In our studies of virtual interferogram, we used Mach-Zehndera and Michelson collected under the scheme. The primary digital hologram was recorded in the laser radiation with a wavelength of $1.064 \mathrm{~mm}, 1.054 \mathrm{~mm}, 0.532 \mathrm{~mm}, 0.357 \mathrm{~mm}$, in pulses with a duration of $30 \mathrm{~ns}$ to $50 \mathrm{ps}$ to $200 \mathrm{fs}$ and emission of continuous He-Ne laser with a digital camera such as the TM-1020-15CL a pixel size $9 \times 9 \mathrm{~mm}$.

The objects used in the shooting: a plate of non-linear optical crystal and $\mathrm{LiNbO}_{3}$ plate photorefractive crystal $\mathrm{LiNbO}_{3}: \mathrm{Fe}(0.01 \%)$. On multicolored holograms recorded by two exposition digital interferometer [6] were obtained digital interferogram different stages of relaxation phase in homogeneities recorded in the volume of the photorefractive crystal $\mathrm{LiNbO}_{3}$ : Fe, as well as the nonlinear optical $\mathrm{LiNbO}_{3}$ crystal. According to the interferograms' recovered phase fronts, supporting quantitative information about the sample was obtained. A comparison of the obtained information - the value of the angle of inclination, a change in the refractive index, the amplitude and length of the surface acoustic waves in various combinations of primary colored phase fronts was performed.

Figure 3 shows a diagram of a recording phase holograms. The radiation of a neodymium laser $\left(\lambda_{1}=1.06 \mathrm{~mm}\right)$ length of $30 \mathrm{~ns}$ and $50 \mathrm{ps}$ with a mirror (1) directed to a non-linear optical crystals $(2,3)$, in which the generation of the second and third harmonics of laser radiation with wavelengths $\lambda_{2}=0.532 \mathrm{~mm}$ and $\lambda_{3}=0.357 \mathrm{~mm}$. 


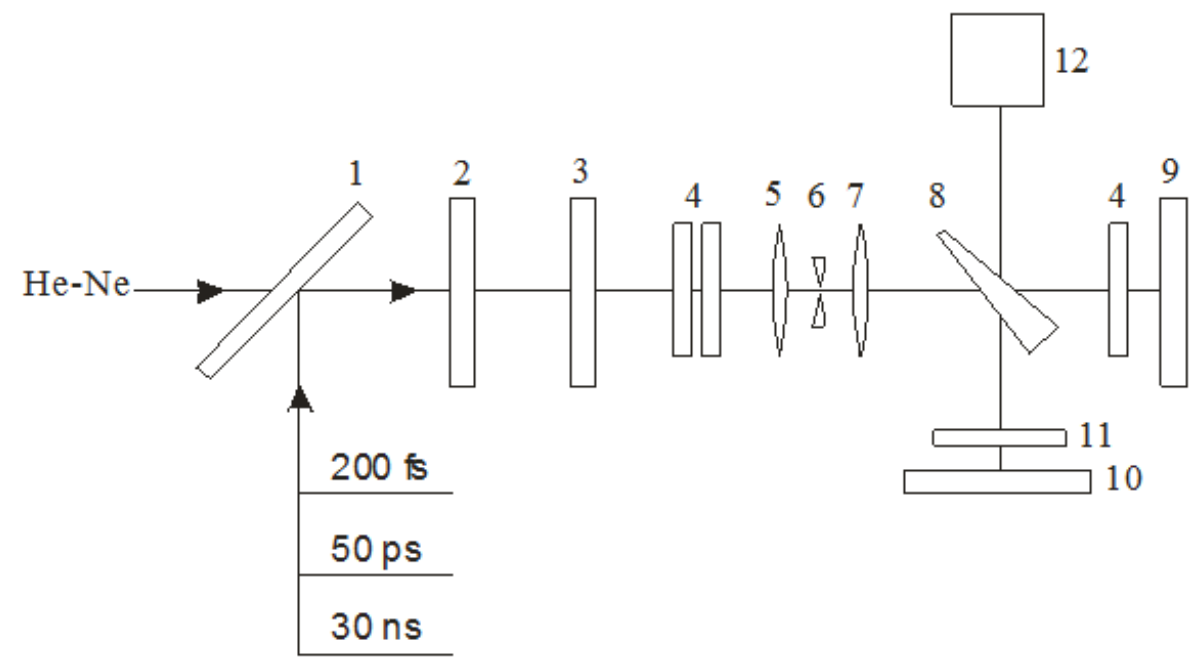

FIG. 3. Scheme of the recording phase holograms: 1 -mirror; 2,3 -nonlinear crystal frequency multiplication; 4 - filters; 5,7 -linza; 6 - spatial filter; 8 - optical wedge; 10 - mirror; 11 - the sample; 12 - CCD camera

Isolation of radiation at a particular wavelength and emission intensity change was carried out by means of filters (4). The emission of He-Ne lasers, at femtosecond levels, would not convert the frequency. Spatial filtering of the radiation was performed by the lenses $(5,7)$ and the diaphragm $(6)$. At the output of the phase front of the spatial filter, the radiation was close to flat. Then, the radiation directed into the interferometer formed by the optical wedge (8) and mirrors $(9,10)$. The sample (11) is located in one of the arms of the interferometer. Register holograms were obtained using a CCD camera (12) connected to a personal computer. Based on the processing of the obtained holograms, the interferogram was constructed.

The resulting phase homogeneity in the laser field in a photorefractive crystal is determined by the intensity of the laser radiation $I(t)$, the exposure time $\tau$, sensitivity photorefractive material $K$ and the absorption coefficient $\alpha$. Fig. 4 shows the calculated phase in homogeneity image in a photorefractive $\mathrm{LiNbO}_{3}: \mathrm{Fe}$ crystals with different exposure times corresponding to the interference pattern in a digital format. The spatial intensity distribution of the laser radiation was supposed to be Gaussian. The exposure time for the image illustrated in Fig. 4b, 3.5 times higher than the exposure time for the image shown in Fig. 4, as well. The figure shows that with increasing exposure times, the phase in the homogeneity image becomes more complex, resulting in increased interference fringes.

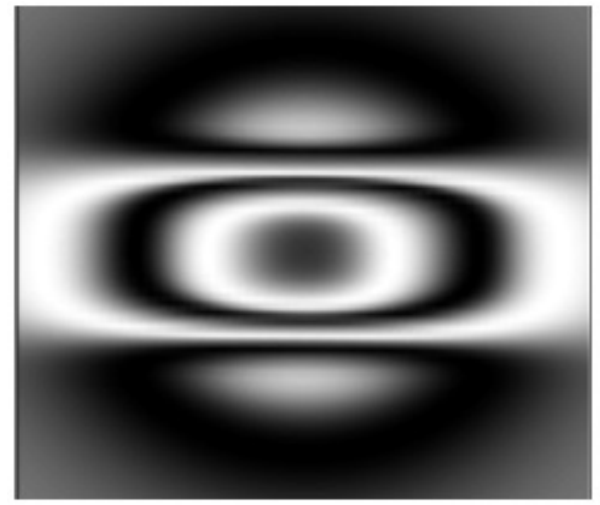

(a)



(b)

FIG. 4. Calculation of photorefractive crystal structure in $\mathrm{LiNbO}_{3}: \mathrm{Fe}$

Figure 5 shows the magnified view of the bulk phase in homogeneity induced in the waist of the focused laser beam, as well as three-dimensional reconstruction phase in homogeneities recorded in $\mathrm{LiNbO}_{3}: \mathrm{Fe}$ radiation with a wavelength of $532 \mathrm{~nm}$. This image is received in the nanosecond laser pulses. The polarization of the 
laser radiation was directed parallel to the optical axis of the crystal. When you rotate the polarization by $90^{\circ}$ pattern has not changed. From the analysis of the recorded phase in homogeneity shown in this figure, it follows that its view qualitatively coincides with the calculated phase distribution (Fig. 4). Some discrepancy may be due to the difference of the beam of laser radiation from the Gaussian. In this case, the main role was played by photorefractive heterogeneity.

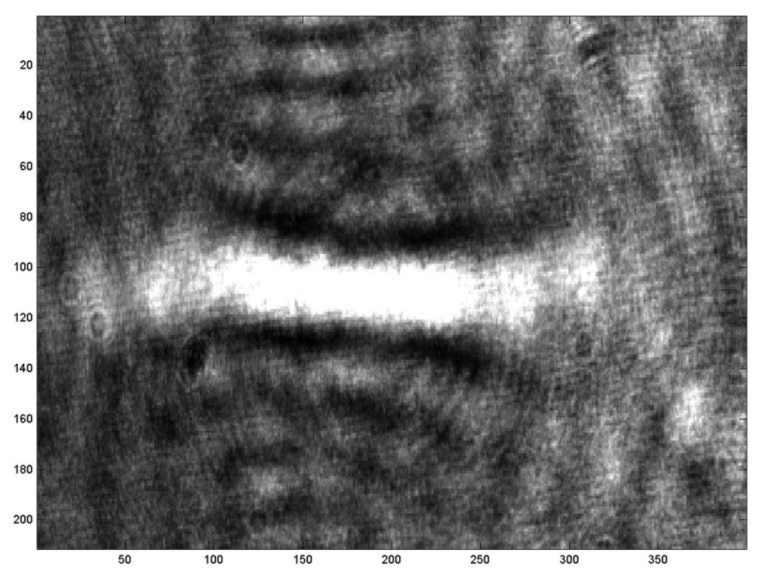

(a)

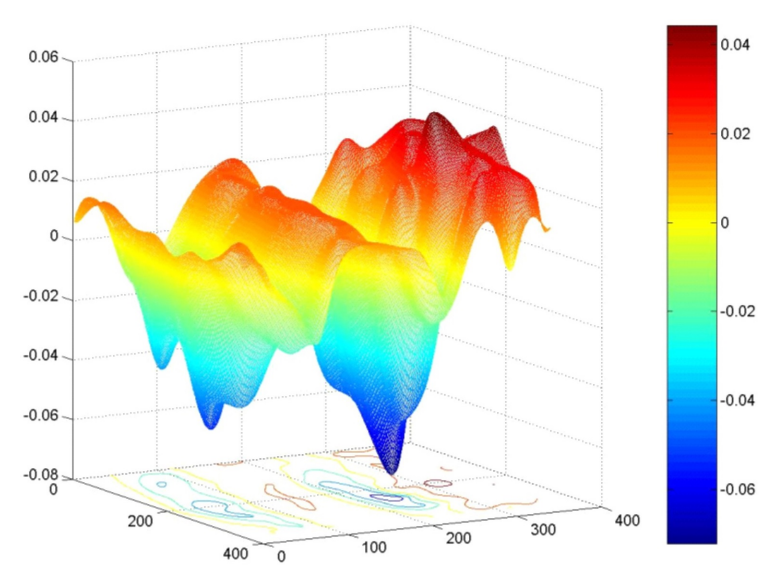

(b)

FIG. 5. Interferogram and phase reconstruction photorefractive structure

In the picoseconds laser phase, change occurs due to the influence of the heterogeneity of the Kerr nonlinearity. Kerr nonlinearity (and hence nonlinear addition to the refractive index) is a tensor quantity that links the components of the third order polarization and the corresponding components of the electromagnetic field. $\mathrm{The}_{\mathrm{LiNbO}}$ nonlinear optical crystal with no loss of Kerr nonlinearity is generally determined by four components [7]. The value of the Kerr nonlinearity depends on the angle of propagation with respect to the main optical axis. To calculate the Kerr nonlinearity values in this case, we used a semi-empirical model. This model is based on taking into account the contribution of the transition in the discrete spectrum.

In this approximation, the Kerr nonlinearity is expressed as a function of the refractive index and its second derivative with respect to wavelength. The expression also includes the density of oscillators $N$, which is defined as the average density of the atoms in the crystal. Fig. 6 shows a simulation of the calculated dependence of the magnitude of the phase shift induced by the intensity of the spatial coordinates at an angle of radiation spread to the main axis of the crystal equal to $72^{\circ}$ at the wavelength $\lambda=532 \mathrm{~nm}$. The initial distribution of the laser intensity was assumed to be Gaussian. The calculations used the following relationship between the components of the non-linearities $\chi_{12}: \chi_{14}: \chi_{23}: \chi_{33}=1: 0.1: 0.2: 6.0$. Note that in this case the components $\chi_{14}$ and $\chi_{23}$ made only a small contribution. From these figures it is clear that the induced nonlinearity has the form of an ellipse, which is approaching the axis of the beam is converted into a dumbbell structure elongated along the $z$-axis of the crystal.

Figure 7, shows a central part of the interferogram fragments obtained by passing the laser radiation with a wavelength of $1.06 \mathrm{~mm} \mathrm{X}$-cut $\mathrm{LiNbO}_{3}$ crystal is $0.2 \mathrm{~cm}$ thick at two different radiation intensities. Radiation polarization direction coincides with the axis $y$. The density of radiation power was $10 \mathrm{GW} / \mathrm{cm}^{2}$.

According to the Fig. 4, the central part of the phase difference expressed in units of wavelength, was $0.085 \mathrm{~mm}$. The calculation showed that the value of the non-linear refractive index $\gamma$ was $1.8 \times 10^{-19} \mathrm{~m}^{2} / \mathrm{W}$. When the crystal orientation of the nonlinear susceptibility of the crystal is determined by the class $3 \mathrm{~m}$ full tensor component $\chi_{12}$, and thus possible to determine the components of the nonlinearity tensor.

We note that for X-cut crystal nonlinear refractive index change may be due to the inverse energy transfer process of the second harmonic of the main emission of laser radiation. Using the known values of the second-order nonlinearity, the refractive index and the correction value were determined by a direct non-linear refractive index, due to the Kerr nonlinearity, which amounted to $1.12 \times 10^{-19} \mathrm{~m}^{2} / \mathrm{W}$.

Similar measurements of the induced optical nonlinearity were performed for other wavelengths and for different crystal orientations with respect to the laser light polarization. Fig. 7b shows a fragment of the central part of an interferogram obtained using the radiation wavelength of the second harmonic of Nd: YAG laser; the nonlinearity size was $\sim 1.4$ times higher than the fundamental nonlinearity frequency. 


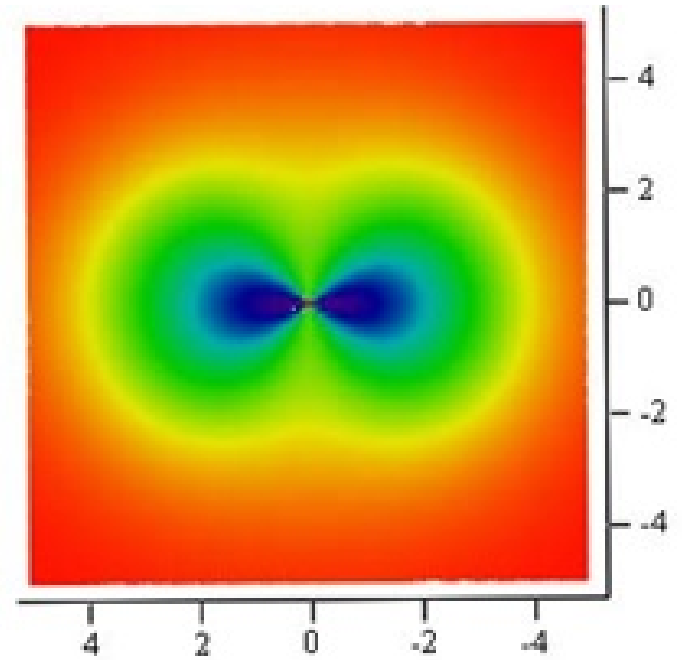

FIG. 6. Calculated dependence of the shift in chip phase difference with the symmetry $3 \mathrm{~m}$, due to the change in the refractive index depending on the intensity of laser radiation

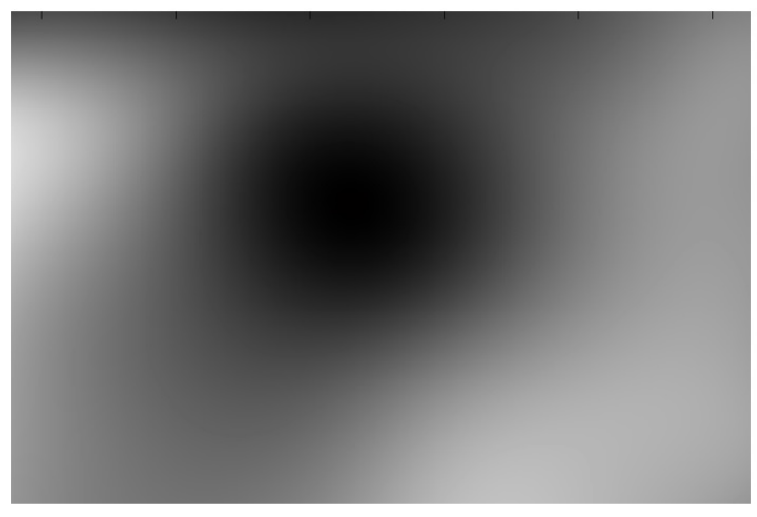

(a)



(b)

FIG. 7. Fragments central interferogram recorded to 1.064 (a) and 0.532 (b) microns

The analysis showed the potential for using a double-exposure digital holographic interferometer for direct measurement of the dynamic emerging in a strong laser field in homogeneity phase and, therefore, made the measurement of non-linear refractive index coefficient photorefractive sensitive material and Kerr nonlinearity perceivable.

Thus, the above study has shown that the digital holographic interferogram obtained using the laser radiation at different wavelengths and durations may possibly be used for the reconstruction of dynamic phase changes occurring in the samples. The most promising practical application of multicolor DHI is the measuring of nanometer-length displacements (nanotechnology, digital microscopy) along with the possibility of using the tunable laser sources in the femtosecond range and the measurement with high temporal resolution, or using cheap laser diodes in the spectral ranges.

\section{References}

[1] Gurevich V.S., Gaponov V.Y., et al. Limit opportunities of a digital holographic interferometry at measurement of midget movements. Control probing devices and automatic equipment in Kazakhstan, 2008, 3 (21), P. 13-14.

[2] Jueptner W., Schnars U. Digital Holography. Springer, Berlin, 2004.

[3] Vizilter Y., et al. Processing and analysis of digital images with examples in LabVIEW and IMAQ Vision. DMK Press, Moscow, 2007.

[4] Cheng Y.-Y., Wyant J.C. Two-Wavelength Phase Shifting Interferometry. Appl Opt., 1984, 23, P. 4639.

[5] Schwider J., et al. Digital wave-front measuring interferometry: Some systematic error sources. Appl. Opt., 1983,22 , P. $3421-3427$.

[6] Redkorechev V.I., et al. Digital picosecond tricolor holographic interferometry. Optics and Spectroscopy, 2009, 107, P. 435-439.

[7] Kulagin I.A. Analysis of third-order nonlinear susceptibilities of quadratic nonlinear optical crystals. J. Opt. Soc. Am. B, 2006, 23, P. $75-80$. 